output $<0.3 \mathrm{ml} / \mathrm{kg} / \mathrm{h}$ for $24 \mathrm{~h}$ or anuria for $12 \mathrm{~h}$; Loss, Failure for $>4$ weeks; End stage, Failure for $>3$ months) 123 patients developed AKI (60 Risk, 32 Injury and 31 Failure), $82 \%$ within 1 week of admission. Baseline characteristics did not differ between children who developed $\mathrm{AKI}$ and those who did not.

Eleven patients required renal replacement therapy (9 Failure and 2 Injury), and 24 died. AKI at admission was associated with increased mortality, independent of admission Pediatric Risk of Mortality (PRISM II) score, and independently predicted duration of stay in both the intensive care unit and hospital. Patients with and without AKI had similar 28-day mortality.

The authors propose that pRIFLE criteria could be used to optimize evaluation and treatment (including initiation of renal replacement therapy) in children with AKI.

Original article Akcan-Arikan A et al. (2007) Modified RIFLE criteria in critically ill children with acute kidney injury. Kidney Int 71: 1028-1035

\section{Graduated fill cycles prevent hydrothorax in children on peritoneal dialysis}

Acute hydrothorax affects $2-4 \%$ of peritoneal dialysis patients. Krishnan et al. have reviewed instances of this complication in 416 children (327 with acute renal failure and 89 with endstage renal failure) who underwent automated in-unit peritoneal dialysis during the 20-year period from June 1985.

Early in the study period, patients with acute renal failure had catheters placed percutaneously and received $40 \mathrm{ml} / \mathrm{kg}$ cycles immediately after placement. The catheters of early enrollees with end-stage renal failure, and those of all patients who joined the study at a later stage, were placed surgically; dialysis fluid volumes in these two groups were increased gradually from $10 \mathrm{ml} / \mathrm{kg}$ to $40 \mathrm{ml} / \mathrm{kg}$ over 6 days.

Overall, 163 patients started on $40 \mathrm{ml} / \mathrm{kg}$ cycles and 253 received the graduated regimen. Acute hydrothoraces developed in 13 children, all of whom had started on $40 \mathrm{ml} / \mathrm{kg}$ cycles. Although 11 of 13 children with hydrothoraces had diarrhea-positive hemolyticuremic syndrome, hydrothorax did not occur in any of the 138 children with this syndrome who had received graduated fills.
In 10 of 12 children aged $\geq 1$ year who developed hydrothoraces, peritoneal dialysis was continued at half-volume with the patient sitting upright; these changes resulted in complete resolution of the hydrothorax over a few days. In six of these patients, cycle volume was increased to $40 \mathrm{ml} / \mathrm{kg}$ without recurrence of hydrothorax; the renal function of the other four children was restored while they were receiving reduced volumes. Two patients were switched to hemodialysis, and one preterm baby died.

The authors conclude that, outside the neonatal period, development of hydrothorax does not necessitate discontinuation of peritoneal dialysis.

Original article Krishnan RG et al. (2007) Acute hydrothorax complicating peritoneal dialysis. Perit Dial Int 27: 296-299

\section{Chronic renal dysfunction after pediatric heart transplantation - risk factors identified}

Long-term immunosuppressive therapy can result in chronic renal insufficiency (CRI). In contrast to the adult population, little is known about the incidence of and risk factors for CRI and end-stage renal disease (ESRD) in the growing number of children who survive following heart transplantation.

Using data from the Scientific Registry of Transplant Recipients, Lee et al. identified all patients younger than 18 years who had received a heart transplant in the US during the period 1990-1999 and survived for more than 1 year. In total, 2,032 patients (median age 4 years) met these inclusion criteria. Mean follow-up was 7 years.

Multivariate analysis revealed pretransplantation hypertrophic cardiomyopathy, African American race, intensive care unit stay at time of transplantation, extracorporeal membrane oxygenation at time of transplantation and preexisting diabetes to be significant risk factors for ESRD. The 5-year actuarial risk for ESRD was $0.9 \%$, rising to $4.3 \%$ at 10 years. The risk of death was sixfold higher in those who developed ESRD than in those who did not.

The incidence of CRI (including ESRD) was examined in 1,055 members of the study population. Multivariate analysis identified pretransplantation dialysis, pretransplantation hypertrophic cardiomyopathy, previous transplantation and 